\section{Alcalóides de Duguetia trunciflora Maas (Annonaceae)}

\author{
I.M. Fechine'; M.A. Lima ${ }^{1}$; V.R. Navarro'; E.V. L. da Cunha ${ }^{1,2}$; \\ M.S. Silva' ${ }^{1}$; J.M. Barbosa-Filho ${ }^{1 *}$; J.G.S. Maia ${ }^{3}$
}

${ }^{1}$ Laboratório de Tecnologia Farmacêutica, Universidade Federal da Paraíba, CP 5009, 58051-970, João Pessoa, PB, Brasil

${ }^{2}$ Universidade Estadual da Paraíba, Departamento de Farmácia e Biologia, Campina Grande, PB, Brasil. 3Departamento de Botânica, Museu Paraense Emílio Goeldi, CP 399, 66040-170, Belém, PA

jbarbosa@ltf.ufpb.br

\begin{abstract}
From the ethanolic extract of the leaves and thin branches of Duguetia trunciflora Maas (Annonaceae) six alkaloids were isolated, being one benzylisoquinoline: reticuline ${ }^{1}$, four tetrahydroprotoberberine: tetrahydro-palmatine ${ }^{2}$, tetrahydrojathrorrizine ${ }^{3}$, discretamine ${ }^{4}$, thaicanine ${ }^{5}$, and one berberine: jathrorrizine ${ }^{6}$. These alkaloids were identified through ${ }^{1} \mathrm{H}$ and ${ }^{13} \mathrm{C}$ NMR spectroscopic technics. The alkaloids $1,3,5$ and 6 are being cited for the first time on Duguetia genus.
\end{abstract}

A família Annonaceae, por apresentar uma combinação de caracteres marcantes, é uma das mais uniformes tanto do ponto de vista anatômico como estrutural e uma das mais primitivas entre as Angiospermae. É constituída por 120 gêneros e aproximadamente 2000 espécies, distribuídas principalmente pelas regiões tropicais do globo. Dos gêneros que compõem esta família, 34 podem ser encontrados na América do Sul, onde predominam os gêneros Annona L, Duguetia St. Hil., Guatteria Ruiz et Pavon, Rollinia St. Hil e Xylopia L. No Brasil, ocorrem cerca de 29 gêneros, incluindo o Duguetia com 50 espécies ${ }^{1}$.

Duguetia trunciflora Maas é uma árvore que pode chegar até $20 \mathrm{~m}$ de altura, apresenta a face inferior da folha esparsamente coberta por escamas estreladas, base obtusa e aguda, com 10-20 nervuras secundárias². Ocorre na Amazônia brasileira, peruana e Guiana Francesa. Muito pouco se sabe a respeito desta planta, pois não há nenhum relato de que seja utilizada na medicina popular e nem tão pouco foi encontrada qualquer citação no Banco de Dados NAPRALERT, Chemical Abstracts e Biological Abstracts. Esta espécie foi coletada na Reserva Mocambo, sob a responsabilidade da EMBRAPA, localizada no perímetro urbano da cidade de Belém-PA (registro: PPDM 393). Este trabalho trata do isolamento e identificação dos constituintes químicos de natureza alcaloidal presentes nas folhas e galhos finos.

O estudo feito com o extrato etanólico de Duguetia trunciflora, tem importância quimiotaxonômica pois mostrou que esta espécie é rica em alcalóides como tem acontecido com outras do mesmo gênero estudadas anteriormente. Um levantamento sobre o gênero Duguetia, feito no Banco de Dados NAPRALERT, Chemical Abstract e Biological Abstracts, mostrou que nas oito espécies descritas na literatura, todas de ocorrência sul americana, foram isolados um total de 79 substâncias, dessas, 72 são alcalóides ${ }^{4}$.

Neste trabalho, foram isolados 6 alcalóides das folhas e galhos finos de D. trunciflora (Figura 1). As substâncias foram identificadas através de métodos espectroscópicos usuais de RMN de ${ }^{1} \mathrm{He}{ }^{13} \mathrm{C}$ (200 e $50 \mathrm{MHz}$ respectivamente) e comparação com dados da literatura ${ }^{5-8}$. Os dados espectroscópicos dos alcalóides isolados de Duguetia trunciflora ${ }^{1-6}$ estão descritos nas Tabelas 1 e 2. Isolou-se um alcalóide benzilisoquinolínico (1), quatro tetrahidroprotoberberínicos (2-4) e um do tipo berberínico (6). Os alcalóides 1, 3, 5 e 6 estão sendo relatados pela primeira vez no gênero Duguetia.

\section{Material e Métodos}

O material coletado, folhas e galhos finos, depois de secos e pulverizados foram extraídos exaustivamente com etanol a $95 \%$. O extrato etanólico foi concentrado à vácuo e em seguida submetido a um tratamento ácido-base para extração dos alcalóides segundo técnica usual ${ }^{3}$. A fração dos alcalóides terciários totais foi submetida à cromatografia em coluna de óxido de alumínio, eluída com misturas de hexano, clorofórmio e metanol em ordem crescente de polaridade. As frações obtidas foram analisadas através de cromatografia em camada delgada analítica, usando como eluente $\mathrm{CHCl}_{3} / \mathrm{MeOH}$ à $2 \%$. Após esse procedimento, as frações que apresentaram os mesmos $\mathrm{Rf}$ foram reunidas e purificadas através de cromatografia em camada delgada preparativa de gel de sílica, resultando no isolamento de seis alcalóides.
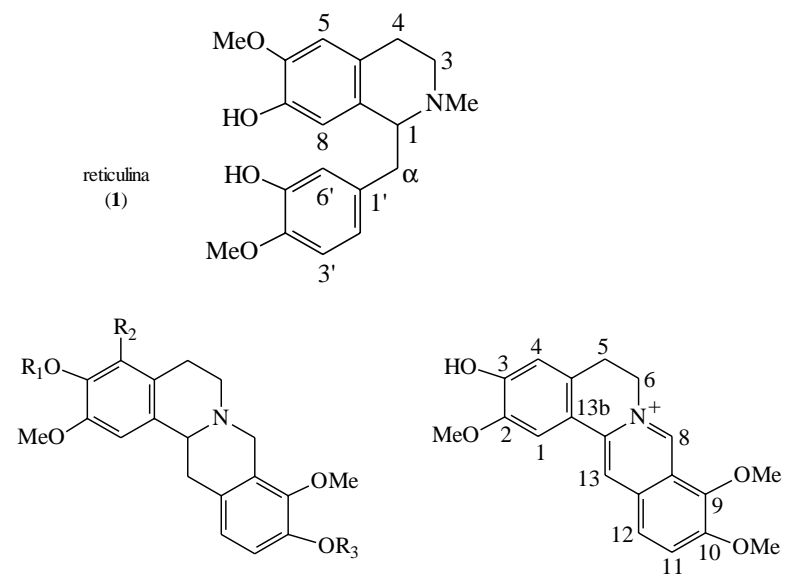

$\begin{array}{ll}\text { (2) } \mathrm{R}_{1}=\mathrm{Me} ; \mathrm{R}_{2}=\mathrm{H} ; \mathrm{R}_{3}=\mathrm{Me} \text { (tetrahidropalmatina) } \\ \text { (3) } \mathrm{R}_{1}=\mathrm{R}_{2}=\mathrm{H} ; \mathrm{R}_{3}=\mathrm{Me} & \text { (tetrahidrojathrorrizina) } \\ \text { (4) } \mathrm{R}_{1}=\mathrm{R}_{2}=\mathrm{R}_{3}=\mathrm{H} & \text { (discretamina) } \\ \text { (5) } \mathrm{R}_{1}=\mathrm{R}_{3}=\mathrm{Me} ; \mathrm{R}_{2}=\mathrm{OH} & \text { (thaicanina) }\end{array}$

jathrorrizina

Figura 1. Alcalóides de Duguetia trunciflora. 
Tabela 1- Deslocamentos químicos de $\mathrm{RMN}^{1} \mathrm{He} \mathrm{e}^{13} \mathrm{C}$ em $\mathrm{CDCl}_{3}$ de (1) isolado de Duguetia trunciflora

\begin{tabular}{l|l|l}
\hline $\mathbf{( 1 )}$ & $\boldsymbol{\delta}^{\mathbf{1}} \mathbf{H}$ & $\boldsymbol{\delta}^{{ }^{13} \mathbf{C}}$ \\
\hline $\mathbf{1}$ & 3,65 & 64,8 \\
\hline $\mathbf{3}$ & $2,75 / 3,15$ & 45,7 \\
\hline $\mathbf{4}$ & $2,55 / 2,80$ & 22,7 \\
\hline $\mathbf{4}$ & - & 124,2 \\
\hline $\mathbf{6}$ & 6,53 & 110,6 \\
\hline $\mathbf{7}$ & - & 146,5 \\
\hline $\mathbf{8}$ & - & 143,2 \\
\hline $\mathbf{8 a}$ & 6,36 & 110,9 \\
\hline $\boldsymbol{\alpha}$ & - & 131,3 \\
\hline $\mathbf{1}$ & $2,80 / 3,00$ & 40,5 \\
\hline $\mathbf{2}$ & - & 127,2 \\
\hline $\mathbf{3}$ & 6,75 & 115,9 \\
\hline $\mathbf{4}$ & - & 145,7 \\
\hline $\mathbf{5}$ & - & 145,9 \\
\hline $\mathbf{6}$ & 6,71 & 114,0 \\
\hline $\mathbf{4}$ '-OMe & 3,83 & 121,3 \\
\hline $\mathbf{6 - O M e}$ & 3,83 & 55,9 \\
\hline $\mathbf{N M e}$ & 2,44 & 55,9 \\
\hline & & 40,6 \\
\hline
\end{tabular}

Tabela 2- Deslocamentos químicos de $\mathrm{RMN}{ }^{13} \mathrm{C}$ e ${ }^{1} \mathrm{H}$ em $\mathrm{CDCl}_{3}$ dos alcalóides 2 a 6 isolados de Duguetia trunciflora

\begin{tabular}{|c|c|c|c|c|c|c|c|c|c|c|}
\hline & \multicolumn{5}{|c|}{$\delta^{13} \mathrm{C}$} & \multicolumn{5}{|c|}{$\delta^{1} \mathbf{H}$} \\
\hline & (2) & (3) & (4) & (5) & (6) & (2) & (3) & (4) & (5) & (6) \\
\hline 1 & 107,7 & 107,8 & 110,6 & 103,1 & 110,6 & 6,75 & 6,67 & 6,64 & 6,37 & 7,58 \\
\hline 3 & 146,6 & 146,7 & 145,2 & 134,3 & 146,6 & - & - & - & - & - \\
\hline 4 & 110,6 & 114,6 & 115,7 & 146,6 & 115,7 & 6,64 & 6,64 & 6,66 & - & 6,98 \\
\hline $4 a$ & 126,9 & 126,8 & 127,8 & 114,3 & 131,7 & - & - & - & - & - \\
\hline $5 \mathrm{eq}$. & 28,7 & 29,6 & 29,7 & 22,6 & 29,7 & 2,67 & 2,67 & 2,51 & 2,58 & 5,60 \\
\hline \multirow{2}{*}{$\begin{array}{l}\text { 6ax. } \\
\text { 6eq. }\end{array}$} & \multirow{2}{*}{51,5} & \multirow{2}{*}{51,4} & \multirow{2}{*}{51,5} & \multirow{2}{*}{51,5} & \multirow{2}{*}{57,6} & 2,67 & 2,69 & 2,59 & 2,84 & 4,90 \\
\hline & & & & & & 3,21 & 3,18 & 3,17 & 3,22 & 5,60 \\
\hline \multirow{2}{*}{$\begin{array}{l}8 \text { 8ax. } \\
\text { 8eq. }\end{array}$} & \multirow{2}{*}{53,8} & \multirow{2}{*}{53,8} & \multirow{2}{*}{53,8} & \multirow{2}{*}{53,8} & \multirow{2}{*}{145,2} & 3,58 & 3,55 & 3,62 & 3,55 & 9,83 \\
\hline & & & & & & 4,26 & 4,23 & 4,62 & 4,25 & - \\
\hline 12 & 124,7 & 124,6 & 124,7 & 124,6 & 124,7 & 6,88 & 6,77 & 6,90 & 6,87 & 7,90 \\
\hline $12 a$ & 128,9 & 128,4 & 129,9 & 128,8 & 128,9 & - & - & - & - & - \\
\hline \multirow{2}{*}{$\begin{array}{l}\text { 13ax. } \\
13 \mathrm{eq} .\end{array}$} & \multirow{2}{*}{36,1} & \multirow{2}{*}{36,1} & \multirow{2}{*}{36,1} & \multirow{2}{*}{36,2} & \multirow{2}{*}{120,9} & 2,84 & 2,83 & 2,97 & 2,84 & 8,82 \\
\hline & & & & & & 3,28 & 3,24 & 3,40 & 3,26 & - \\
\hline $13 \mathbf{a}$ & 59,4 & 59,4 & 59,5 & 59,4 & 135,1 & 3,55 & 3,10 & 3,56 & 3,51 & - \\
\hline $13 b$ & 129,9 & 128,8 & 129,9 & 134,3 & 121,0 & - & - & - & - & - \\
\hline 2-OMe & 55,8 & 55,8 & 56,1 & 55,8 & 59,4 & 3,85 & 3,86 & 3,85 & 3,84 & 3,82 \\
\hline 3-OMe & 55,8 & - & - & 60,4 & - & 3,86 & - & - & 3,85 & - \\
\hline 9-OMe & 60,5 & 60,1 & 59,5 & 60,4 & 63,0 & 3,87 & 3,83 & 3,87 & 3,87 & 3,91 \\
\hline
\end{tabular}




\section{Agradecimentos}

Os autores agradecem ao Banco de Dados NAPRALERT, da Universidade de Illinois, USA, pelo levantamento bibliográfico e ao CNPq e CAPES pelo apoio financeiro.

\section{Refrências}

${ }^{1}$ Hutchinson J. The genera of flowering plants. Clarendon Press, Oxford, 1964. p. 71-108

${ }^{2}$ Ribeiro JEL. Flora da Reserva Duck: Guia de identificação das plantas vasculares de uma floresta de terra firme na Amazônia Central. INPA, Manaus, 1999. 816p

${ }^{3}$ Sette IMF, Da-Cunha EVL, Barbosa-Filho JM, Silva MS. Tetrahydroprotoberberine and aporphine alkaloids from Rollinia leptopetala. Pharmaceutical Biology 2000; 38: 318320

${ }^{4}$ Navarro VR, Sette IMF, Da-Cunha EVL, Silva MS, BarbosaFilho JM, Maia JGS. Alcalóides de Duguetia flagellaris Huber (Annonaceae). Revista Brasileira de Plantas Medicinais 2001 (No prelo)

${ }^{5}$ Hussain AR, Kim J, Beecher WWC, Kinghprn DA. Unambiguous carbon-13 NMR assignments of some biologicaly active protoberberine alkalois. Heterocycles 1989; 29: 2257-2260

${ }^{6}$ Bhakuni DS, Gupta S. The alkaloids of Stephania glabra. J. Nat. Prod. 1982; 45: 406-411

${ }^{7}$ Chang HM, El-Fishawy AM, Slatkin DJ, Schiff-Junior PL. Quaternary alkaloids of Tinospora capillipes. Planta Medica 1984; 88-90

${ }^{8}$ Ohiri FC, Verpoorte R, Baerheim-Svendsen A. ${ }^{1} \mathrm{H}$ NMR chemical shifts values for aromatic prótons in 2, 3, 9, 10- and 2, 3, 10, 11tetrasubstituted tetrahydroprotoberberine alkaloids. Planta Medica 1983; 49: 162-164

\section{Extração com solvente e fluido supercrítico dos constituintes do caule subterrâneo de Spiranthera odoratissima A. St.-Hil. (Rutaceae)}

\section{Clécia Maria de Jesus Freitas ${ }^{1}$; Maria Lenise da Silva

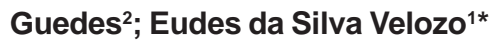

'Laboratório de Pesquisa em Matéria Médica (LAPEMM), Faculdade de Farmácia

2Instituto de Biologia

Universidade Federal da Bahia, 40.170-290, Salvador, BA, Brasil

lapemm@ufba.br

\section{Resumo}

$\mathrm{O}$ arbusto Spiranthera odoratissima (Rutaceae) foi coletado no município de Mucugê (BA). Seu caule subterrâneo foi submetido a extração com solvente orgânico e fluido supercrítico. A extração com $\mathrm{CO}_{2}$ supercrítico forneceu a 8-prenil7-geraniloxicumarina. A partir do extrato $\mathrm{CH}_{2} \mathrm{Cl}_{2}$ isolou-se a cumarina aurapteno e identificou-se o alcalóide esquimianina. Estas substâncias foram identificadas com base na análise dos seus espectros de RMN ${ }^{1} \mathrm{H}$ e ${ }^{13} \mathrm{C}$, IV e comparação com dados da literatura.

\begin{abstract}
Supercritical fluid and solvent extraction of rhizome from Spiranthera odoratissima A. St.-Hil. (Rutaceae).

The shrub S. odoratissima was collected at Mucugê (Chapada Diamantina - Bahia, Brazil). Its rhizome was extracted by maceration with $\mathrm{CH}_{2} \mathrm{Cl}_{2}$ and supercritical $\mathrm{CO}_{2}$. The supercritical extraction supplied a coumarin, 8-prenyl7 geranyloxycoumarin. Another coumarin, the auraptene, and an alkaloid, skimmianine, were obtained from the $\mathrm{CH}_{2} \mathrm{Cl}_{2}$ extract. The structures of the compounds were elucidated based on spectroscopic studies, and by comparison with literature data.
\end{abstract}

A medicina popular tem profundas raízes na cultura do povo nordestino alicerçada nos conhecimentos dos negros, trazidos como escravos durante os séculos XVI a XIX, fundido com o saber dos nativos. Esta farmacopéia tem mostrado-se uma fonte valiosa de informações para o conhecimento e aplicação terapêutica da flora regional. A planta conhecida como acabadeira ou sarrinha, na região da Chapada Diamantina (BA), e manacá no estado do Mato Grosso, tem suas folhas e caules subterrâneos utilizados, na forma de decocto no vinho ou na cachaça, no tratamento de reumatismo, gota, infecções nos rins e inflamações em geral, tendo ainda ação diurética e depurativa. As informações tradicionais relatam que seu consumo em ex- 